\title{
Carotid artery Doppler ultrasonography in retinal macroaneurysms
}

\begin{abstract}
It is postulated that retinal arterial macroaneurysms (RAMs) occur at the site of incomplete embolic occlusion of a branch retinal artery. Embolic events of the retinal vessels are related to the state of the carotid artery tree and therefore Doppler ultrasonography of the carotid arteries in these patients is of particular interest. We have examined 13 patients with retinal artery macroaneurysms with carotid artery Doppler ultrasonography (CADU). Eight of these patients (61.5\%) exhibited atheromatous plaques on the same side as the RAM with a moderate degree of arterial narrowing. Although our group of patients did not show advanced carotid artery disease, alterations of the arterial wall found at the level of the carotid artery were higher than expected in a similar hypertensive population. To our knowledge this is the first study of the carotid arterial tree in these patients. Our results support the theory that RAMs may be of embolic origin. Furthermore they demonstrate that CADU is a useful investigation in patients with RAMs.
\end{abstract}

Key words Carotid artery, Retinal macroaneurysms, Doppler ultrasonography

Retinal artery macroaneurysms (RAMs) were first described in a published report in 1880 by Loring, in which he described a peculiar bulging within the arterial tree. ${ }^{1}$ Robertson in 1973 first applied the term macroaneurysm to saccular retinal artery dilatations sited on the first three orders of the arterial tree. ${ }^{2}$ These were found mainly at arterial bifurcations. RAMs can present due to visual loss from macular involvement or breakthrough vitreous haemorrhage but may remain asymptomatic. They occur most commonly in the temporal vessels and $50 \%$ have an associated retinal haemorrhage. Subretinal, preretinal or vitreous haemorrhages may be seen in association with intraretinal haemorrhages. RAMs may also be exudative causing macular oedema and lipid deposition, although haemorrhage and exudate
G. THURAIRAJAN, T. POTAMITIS,

G. NAYLOR, J. GIBSON may coexist. ${ }^{1,2}$ In the majority of cases RAMs resolve spontaneously and good visual function is retained. ${ }^{2,3}$ In the event of recurrent vitreous haemorrhage or persisting macular oedema or exudate, laser treatment is required. Laser treatment may be applied directly to the RAM, or indirectly to the surrounding retina using argon or yellow dye photocoagulation. ${ }^{1-4}$

The precise pathogenesis of arterial macroaneurysms is not known. They are structurally similar to the intracerebral miliary aneurysms occurring in elderly hypertensive patients. Lewis et al. ${ }^{5}$ documented the development of macroaneurysms from the site of an incomplete embolic occlusion of a branch retinal artery. RAM formation from the exact site of a previous retinal artery embolus has been described. ${ }^{6}$ It is postulated that local vessel wall damage from emboli or local thrombosis predisposes to aneurysmal dilatation. As embolic events in the retinal arterial network are closely associated with the state of the carotid arterial tree, Doppler ultrasonography of the carotid arteries in these patients is of particular interest. We have therefore routinely performed carotid artery Doppler ultrasonography (CADU) on all patients with RAMs. We report here the findings and visual outcome of 13 patients presenting with RAMs who had CADU performed.

\section{Patients and methods}

Thirteen patients presenting to our Macular Clinic with RAMs (8 female, 5 male) were included in the study. All 13 patients underwent CADU by the same consultant radiologist. The mean age of the patients was 74 years (range 57-88 years). Information regarding mode of presentation, visual acuity, general medical history and treatment was obtained from the clinical records. All patients were then reviewed, had final best corrected visual acuity measured and underwent dilated fundoscopy by one of the authors. All patients had their blood pressure checked at presentation and blood specimens were obtained for a full blood count, ESR and a lipid profile.
G. Thurairajan
T. Potamitis
G. Naylor
J. Gibson
Birmingham and Midland
Eye Centre
City Hospital NHS Trust
Dudley Road
Birmingham B187QH, UK 
Table 1. Patient details

\begin{tabular}{ll}
\hline Age (years) & Range $57-88$, mean 73.8 \\
Sex & $61.5 \%(n=8)$ female $/ 38.5 \%(n=5)$ male \\
Laterality & All unilateral \\
Presentation & $38.5 \%(n=5)$ haemorrhagic \\
& $15.4 \%(n=2)$ vitreous haemorrhage \\
& $46.1 \%(n=6)$ exudative \\
Vision on presentation & Range $6 / 6$ to $C F$ \\
& $54 \%(n=7) 6 / 36$ or worse \\
Vision on resolution & Range $6 / 6$ to $\mathrm{CF}$ \\
& $53.8 \%(n=7)$ better than $6 / 36$ \\
& $46.1 \%(n=6) 6 / 12$ or better \\
Laser treatment & $38.5 \%(n=5)$ patients received treatment for persisting macular oedema or exudate \\
Hypertension & $70 \%(n=9)$ were on hypertensive medication \\
Carotid artery Doppler scanning & $61.5 \%(n=8)$ exhibited atheromatous plaques and a moderate degree of arterial narrowing. \\
& Six patients with abnormal scans and three with normal scans were hypertensive \\
\hline
\end{tabular}

\section{Results}

The clinical characteristics of our 13 patients are summarised in Table 1. On ophthalmic examination all RAMs were unilateral. Seven patients $(53.9 \%)$ presented with a haemorrhagic RAM, of which 2 had a secondary vitreous haemorrhage; 6 patients (46.1\%) presented with an exudative RAM. Visual acuity at presentation was worse than $6 / 36$ in 7 patients $(53.9 \%$ ) with a range of $6 / 6$ to count fingers. Five patients $(38.5 \%)$ required laser treatment for persistent macular oedema or exudate.

Nine patients $(70 \%)$ were on anti-hypertensive medication and 2 were found to have hyperlipidaemia. Two patients suffered from non-insulin-dependent diabetes mellitus and one patient had a previous history of myocardial infarction.

CADU was performed in all 13 patients. Eight patients $(61.5 \%)$ exhibited a moderate degree of arterial narrowing and atheromatous plaques on the same side as the RAM. None of the patients demonstrated greater than $50 \%$ narrowing on CADU. Six of the patients with abnormal scans and 3 of the patients with normal scans were on treatment for hypertension. On resolution of the RAM visual acuities ranged from $6 / 6$ to count fingers, with $7(53.8 \%)$ patients having a vision of $6 / 36$ or better.

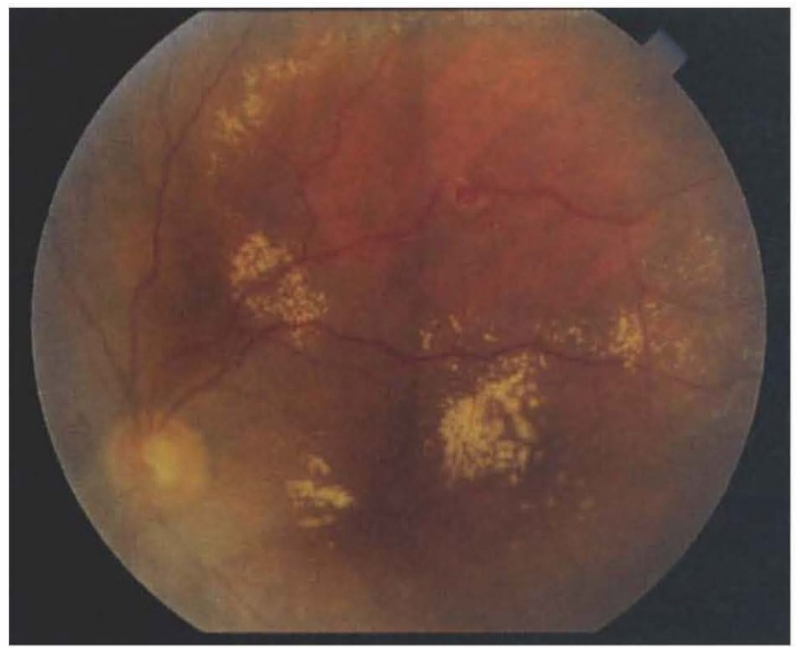

Fig. 1. An exudative retinal artery macroaneurysm.

\section{Discussion}

The clinical characteristics of RAMs have been well documented and are reproduced in our series. RAMs are seen in the elderly and rarely before the age of 60 years. There is a marked female predominance and they are typically unilateral. Arteriosclerosis has been suggested as a causal factor and systemic hypertension is the most commonly associated disease at the time of diagnosis, with up to $75 \%$ of patients with RAMs being hypertensive. Diabetes mellitus and the hyperlipidaemias are also common associations. ${ }^{1}$ The risk of a cerebrovascular accident or myocardial infarction has been found to be greater than $25 \%$ within 5 years of presentation with a RAM. ${ }^{5}$ This emphasises the fact that the presence of a RAM does indicate coexisting systemic disease and the need for appropriate management.

To our knowledge this is the first study of the carotid arterial tree in patients presenting with RAMs. The increased incidence of atheromatous change in the elderly hypertensive population is well documented. Roman et al. ${ }^{7}$ looked at the carotid intimal medial thickness in the normotensive and asymptomatic hypertensive population. Carotid wall thickness was documented in excess of the 95 th percentile in $28 \%$ of the

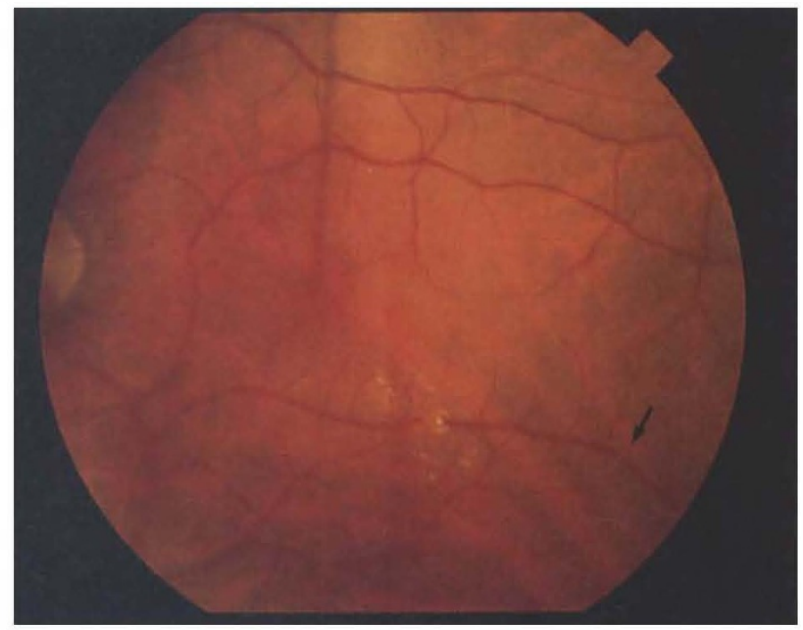

Fig. 2. A retinal artery macroaneurysm with a distal embolus (arrow) in the same vessel, which supports our theory (see text). 
hypertensive population. Routine screening with CADU of asymptomatic patients with peripheral vascular disease has a significant pick-up rate. Forty-seven per cent of these patients were found to have some degree of carotid artery disease though there was no audible carotid bruit. Furthermore on looking at the patients over the age of 68 years with one or more of the other risk factors such as male gender, hypertension, diabetes mellitus, and previous or recent vascular disease, the incidence of advanced carotid artery disease (greater than $50 \%$ narrowing) was $45 \%{ }^{8}$ The presence or absence of a carotid bruit is not always a reliable indicator of the presence of carotid vascular disease. Advanced disease may completely occlude the artery. This would eliminate the risk of an embolism and since there could be no flow a bruit would not be audible. It follows that a loud bruit would indicate adequate blood flow through the vessel thereby facilitating embolisation of atheromatous material distal to the carotid arterial wall abnormalities.

Though our group of patients did not exhibit advanced disease, the incidence of carotid arterial wall abnormalities was higher than that found in a similar asymptomatic hypertensive population. ${ }^{7}$ This also supports the theory that RAMs are of embolic origin. Carotid atheromatous disease is the commonest cause of acute and chronic obstruction of the ocular arterial circulation. In the United Kingdom CADU is recommended in patients with a history of transient ischaemic attacks, retinal infarction, amaurosis fugax or the ocular ischaemic syndrome. It is our view that RAMs are also an indication for CADU. Several studies have shown that surgical intervention is only of benefit to patients with severe carotid artery stenosis. ${ }^{9-11}$ Milder forms of carotid artery sclerosis, however, tend to be a manifestation of wider arterial disease. ${ }^{8}$ It has been shown that carotid artery disease is positively correlated with coronary artery disease and left ventricular hypertrophy. ${ }^{12,13}$ Cardioembolic sources must also be considered in the pathogenesis of RAMs. This emphasises the need for appropriate medical intervention. Lipid-lowering drugs such as pravastatin and lovastatin and mini-dose warfarin may play a role in the management of patients with asymptomatic carotid vascular disease. ${ }^{14,15}$ Systemic investigation and referral to a physician for a full medical investigation to identify and treat other risk factors may be of benefit in patients with RAMs.

\section{References}

1. Rabb MF, Gagliano DA, Teske MP. Retinal artery macroaneurysms. Surv Ophthalmol 1988;33:73-96.

2. Abdel-Khalek MN, Richardson J. Retinal macroaneurysm: natural history and guidelines for treatment. $\mathrm{Br} \mathrm{J}$ Ophthalmol 1986;70:2-11.

3. Brown DM, Sobol WM, Folk JC, Weingeist TA. Retinal arteriolar macroaneurysms: long-term visual outcome. $\mathrm{Br} \mathrm{J}$ Ophthalmol 1994;78:534-8.

4. Joondeph BC, Joondeph HC, Blair NP. Retinal macroaneurysms treated with the yellow dye laser. Retina 1989;9:187-92.

5. Lewis RA, Norton EWD, Gass JDM. Acquired arterial macroaneurysms of the retina. Br J Ophthalmol 1976;60:21-30.

6. Wiznia RA. Development of a retinal artery macroaneurysm at the site of a previously detected retinal artery embolus. Am J Ophthalmol 1992;114:642-3.

7. Roman MJ, Saba PS, Pini R, Spitzer M, Pickering TG, Rosen S, et al. Parallel cardiac and vascular adaptation in hypertension. Circulation 1992;86:1909-17.

8. Ahn SS, Baker JD, Walden K, Moore WS. Which asymptomatic patients should undergo routine screening carotid duplex scan? Am J Surg 1991;162:180-5.

9. European Carotid Surgery Trialists Collaborative Group. Interim results for symptomatic patients with severe (70$99 \%$ ) or with mild (0-29\%) carotid stenosis. MRC European carotid surgery trial. Lancet 1991;337:1235-43.

10. North American Symptomatic Carotid Endarterectomy Trial (NASCET) Collaborators. Beneficial effect of carotid endarterectomy in symptomatic patients with high grade stenosis. N Engl J Med 1991;325:445-53.

11. The CASANOVA Study Group. Carotid surgery versus medical therapy in asymptomatic carotid stenosis. Stroke 1991;22:860.

12. Chambers BR, Norris JW. Outcome in patients with asymptomatic neck bruits. N Engl J Med 1986;315:860-5.

13. Roman MJ, Pickering TG, Schwartz JE, Pini R, Devereux RB. Association of carotid atherosclerosis and left ventricular hypertrophy. J Am Coll Cardiol 1995;25:83-90.

14. Byrington RP, Fuberg CD, Crouse JR 3rd, Espeland MA, Bond MG. Pravastatin, lipids and atherosclerosis in the carotid arteries (PLAC-II). Am J Cardiol 1995;76:C54-9.

15. Furberg CD, Adams HP Jr, Applegate WB, Byington RP, Espeland MA, Hartwell T, et al. Effect of Lovastatin on early carotid ateriosclerosis and cardiovascular events. Circulation 1994;90:1679-88. 